\title{
Harlequin baby- case of birth of a monster baby! - A myth!
}

\author{
Shweta $^{1} *$, Neelima Singh ${ }^{2}$
}

\author{
${ }^{1}$ Department of Obstetrics and Gynecology, MGMCRI, SBV University, Pondicherry, India \\ ${ }^{2}$ Department of Obstetrics and Gynecology, PIMS, Pondicherry, India
}

Received: 3 June 2013

Accepted: 15 June 2013

\section{*Correspondence:}

Dr. Shweta,

E-mail: Shwetaguptadr15@gmail.com

(C) 2013 Shweta et al. This is an open-access article distributed under the terms of the Creative Commons Attribution Non-Commercial License, which permits unrestricted non-commercial use, distribution, and reproduction in any medium, provided the original work is properly cited.

\begin{abstract}
Harlequin phenotype is inherited as autosomal recessive trait. Disease has been known since 1750, atleast 100 cases have been reported worldwide in modern times. Many cases are sporadic and many others occur in consanguineous families where more than one child is affected. We report a case of harlequin baby born to a mother who had previous two similar babies and two other normal babies.
\end{abstract}

Keywords: Harlequin, Autosomal recessive

\section{INTRODUCTION}

Harlequin ichthyosis should be regarded as a severe chronic disease that is not invariably fatal. With improved neonatal care and probably the early introduction of oral retinoids, the number of survivors is increasing. ${ }^{4}$ Those from families with a history of severe skin disorders may have a higher risk of birthing a harlequin child. ${ }^{1}$

The survival period of these babies is variable, most succumb to illness within $24-48$ hrs \& others usually die within one week of life. Those who survive have severe ichthyosis and variable neurological impairment. Defects in keratin expression and epidermal lipid deposition, keratinocytes show excessive cornification, failure of desquamation. ${ }^{1,2}$

Abnormal lipid metabolism in mitochondria has been suggested as the cause of defective lamellar body formation.

\section{CASE REPORT}

A 35 year old unbooked $5^{\text {th }}$ gravida was admitted in emergency hours at SSH, BHU Varanasi at 38 weeks of gestation with diminished fetal movements for 4 days. Past obstetric history of the patient revealed that she had two surviving normal female babies and she had lost two male babies who had abnormal appearances and both babies had died after one day of birth.

The general examination of the patient revealed no abnormality, on per abdominal examination - uterus was term size with cephalic presentation with contractions \& FHR 90-100bpm. Routine antenatal investigations were sent. Patient was immediately shifted for emergency LSCS in view of fetal distress. A Harlequin baby (M 2.8 $\mathrm{kg}$ ) was delivered, the cry of the baby was feeble.

The whole body of the baby was encased in a rigid hyperkeratotic 'coat of armour'. The whole skin was cracked and ridged, the facial features were distorted. The eyes were red, severe chemosis was present, eyeballs could not be seen. Bilateral ectropion was present. The nose was absent, two pits seen in its place. The external ears were missing only the pits could be seen. The mouth was big and wide open. The gums were prominent and lips were everted. The hands and feet were swollen and rigid, the nails were absent There were few hair on the back of the head. The external genitalia present but covered with hyperkeratotic skin, the joint mobility was restricted all over due to hyperkeratosis. The respiration of the baby was depressed, the sucking was poor. 


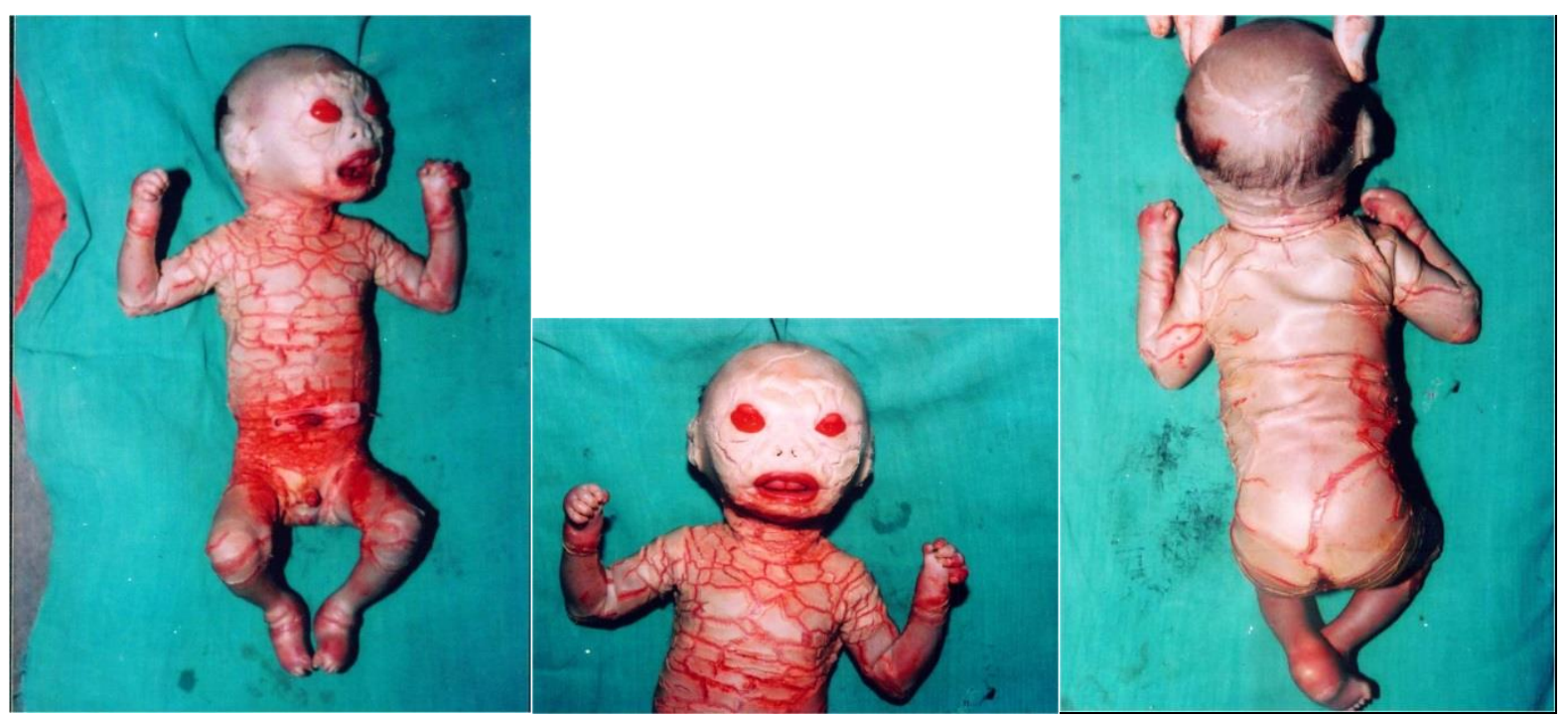

\section{DISCUSSION}

In our case baby delivered had features of harlequin ichthyosis. If this patient had come to us at earlier gestation, then certain set of investigations following conception could have resulted in earlier diagnosis.

Diagnosis is done by amniocentesis at 17 weeks shows intracellular lipid vesicles in dumped shed keratinocytes presumably derived from follicular epidermis, fetal skin biopsy done at $18^{\text {th }}$ week. Common features of the disease through ultrasound, and follow up with 3D ultrasound can diagnose the condition.

Treatment consists of supportive care in a humid, temperature controlled environment frequent application of topical emollients to skin to keep the skin supple. Use of oral retinoids. Careful attention to hygiene. Complications of such babies are sepsis, inability to feed leading to hypoglycemia, dehydration and renal failure, temperature instability, inadequate ventilation. 1,5

\section{REFERENCES}

1. James, William; Berger, Timothy; Elston, Dirk (2005). Andrews' Diseases of the Skin: Clinical Dermatology. (10th ed.). Saunders.

2. Kelsell DP, Norgett EE, Unsworth H, et al. (May 2005). Mutations in ABCA12 underlie the severe congenital skin disease harlequin icthyosis. Am. J. Hum. Genet.76 (5):794-80.

3. Alison Jones (May 9, 2008). Nelly is a real diamond girl. Brimingham post. Retrieved 2008-11-10.

4. Rajpopat S, Moss C, Mellerio J, Vahlquist A, Gånemo A, Hellstrom-Pigg $\mathrm{M}$, et al. Harlequin ichthyosis: a review of clinical and molecular findings in 45 cases. Arch Dermatol. 2011 Jun;147(6):681-6.

5. Waring JJ. Early Mention of a Harlequin Fetus in America. Am J Dis Child 1932;43:442.

DOI: $10.5455 / 2320-1770 . i j r \operatorname{cog} 20130941$

Cite this article as: Shweta, Singh N. Harlequin baby- case of birth of a monster baby! - A myth! Int J Reprod Contracept Obstet Gynecol 2013;2:447-8. 